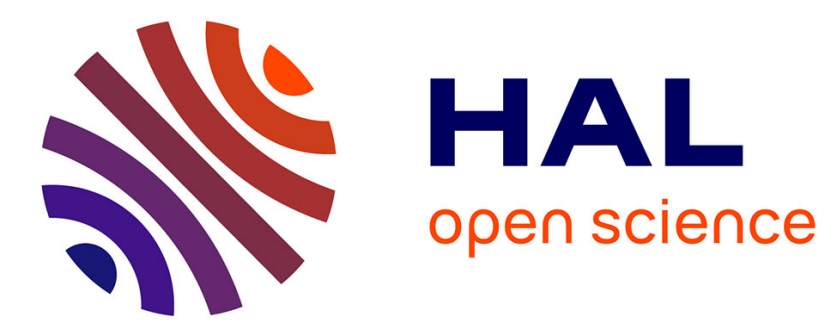

\title{
On Moment Problems with Holonomic Functions
}

Florent Bréhard, Mioara Joldes, Jean-Bernard Lasserre

\section{To cite this version:}

Florent Bréhard, Mioara Joldes, Jean-Bernard Lasserre. On Moment Problems with Holonomic Functions. 44th International Symposium on Symbolic and Algebraic Computation (ISSAC 2019), Jul 2019, Pékin, China. pp.66-73. hal-02006645

\section{HAL Id: hal-02006645 \\ https://hal.science/hal-02006645}

Submitted on 4 Feb 2019

HAL is a multi-disciplinary open access archive for the deposit and dissemination of scientific research documents, whether they are published or not. The documents may come from teaching and research institutions in France or abroad, or from public or private research centers.
L'archive ouverte pluridisciplinaire HAL, est destinée au dépôt et à la diffusion de documents scientifiques de niveau recherche, publiés ou non, émanant des établissements d'enseignement et de recherche français ou étrangers, des laboratoires publics ou privés. 


\title{
On a moment problem with holonomic functions
}

\author{
Florent Bréhard ${ }^{1}$, Mioara Joldes ${ }^{2}$, and Jean-Bernard Lasserre ${ }^{3}$ \\ ${ }^{1}$ ENS de Lyon \& LAAS-CNRS, Lyon \& Toulouse, France, \\ florent.brehard@ens-lyon.fr \\ ${ }^{2}$ LAAS-CNRS, Toulouse, France, joldes@laas.fr \\ ${ }^{3}$ LAAS-CNRS, Toulouse, France, lasserre@laas.fr
}

\begin{abstract}
Many reconstruction algorithms from moments of algebraic data were developed in optimization, analysis or statistics. Lasserre and Putinar proposed an exact reconstruction algorithm for the algebraic support of the Lebesgue measure, or of measures with density equal to the exponential of a known polynomial. Their approach relies on linear recurrences for the moments, obtained using Stokes theorem.

In this article, we extend this study to measures with holonomic densities and support with real algebraic boundary. In the framework of holonomic distributions (i.e. they satisfy a holonomic system of linear partial or ordinary differential equations with polynomial coefficients), an alternate method to creative telescoping is proposed for computing linear recurrences for the moments. When the coefficients of a polynomial vanishing on the support boundary are given as parameters, the obtained recurrences have the advantage of staying linear with respect to them.

This property allows for an efficient reconstruction method. Given a finite number of numerically computed moments for a measure with holonomic density, and assuming a real algebraic boundary for the support, we propose an algorithm for solving the inverse problem of obtaining both the coefficients of a polynomial vanishing on the boundary and those of the polynomials involved in the holonomic operators which annihilate the density.
\end{abstract}




\section{Introduction}

Notations. Let $n$ be a positive integer for the ambient space $\mathbb{R}^{n}$, whose canonical basis is denoted by $\left(\boldsymbol{e}_{\boldsymbol{1}}, \ldots, \boldsymbol{e}_{\boldsymbol{n}}\right)$. Let $\mathbb{K}[x]$ be the ring of polynomials in the variables $x=\left(x_{1}, \ldots, x_{n}\right)$ over a real finite computable extension of $\mathbb{Q}$, and let $\mathbb{K}[x]_{d}$ be the vector space of polynomials of total degree at most $d$. For every $d$, let $\mathbb{N}_{d}^{n}:=$ $\left\{\alpha \in \mathbb{N}^{n}:|\alpha| \leqslant d\right\}$, where $|\alpha|=\sum_{i} \alpha_{i}$. In a multivariate setting, we denote $x^{\beta}=x_{1}^{\beta_{1}} \ldots x_{n}^{\beta_{n}}$ and $\partial_{x}^{\alpha}=\partial_{x_{1}}^{\alpha_{1}} \ldots \partial_{x_{n}}^{\alpha_{n}}$ for $\alpha, \beta \in \mathbb{N}^{n}$. The derivative $\frac{\partial p}{\partial x_{i}}$ is denoted $p_{x_{i}}$. The indicator function of a set $G$, is denoted by $\mathbb{1}_{G}$.

The structure of moments of algebraic data is a central question in various reconstruction algorithms, appearing as part of a broad field of inverse problems [9]. We refer to [14] and references therein for various shape reconstruction from their moments of polyhedra [7, 6], planar quadrature domains [4], sublevel sets of homogeneous polynomials [13], together with more applied studies of computerized tomography [16].

In this article, we focus on the structure of moments of holonomic distributions, together with associated inverse problems. It can be seen as a computer algebra-based extension of [14], where the approach was mainly based on techniques recently developed in polynomial optimization [12], which are at the interface between real algebraic geometry, moment problems and polynomial optimization. More precisely, our setting is the following.

Setting: Let $G \subset \mathbb{R}^{n}$ be a bounded open set of Euclidean space, whose boundary $\partial G$ is algebraic ( $\partial G$ is contained in the real zero set of finitely many polynomials), and let $\mu_{f}=f \mathbb{1}_{G} \mathrm{~d} x$ be a measure supported on $G$, with a so-called holonomic weight $f$ against Lebesgue volume measure $\mathrm{d} x$ on $\mathbb{R}^{n}$. This means that it satisfies a holonomic system of linear partial or ordinary differential equations with polynomial coefficients (as a generalized function if needed, see Def. 3). Consider also the power moments of $\mu_{f}$ :

$$
m_{\alpha}=\int_{G} x^{\alpha} \mathrm{d} \mu_{f}(x), \quad \alpha \in \mathbb{N}^{n} .
$$

For instance, the weight $f(x)=\exp (p(x))$, with $p \in \mathbb{R}[x]_{d}$ is holonomic i.e., it satisfies:

$$
\left\{\frac{\partial f}{\partial x_{i}}-\frac{\partial p}{\partial x_{i}} f=0, \quad i=0, \ldots, n .\right.
$$

In [14], the following property is proved, for such an exponential-polynomial weight: knowing a priori the coefficients of $p$, its degree $s$ and the degree $d$ of the variety containing $\partial G$, a threshold $N$ is identified (which depends only on $d$ and $s$ ), such that the moments $m_{\alpha}$ up to degree $N$ (i.e. $\alpha \in \mathbb{N}_{N}^{n}$ ) determine 
in a constructive and robust manner the coefficients of a polynomial vanishing on $\partial G$.

A natural question is whether this result can be generalized, as mentioned in [14]: the analogy to the well understood moment rigidity of the Gaussian distribution is striking, although the constructive aspects of this finite determinateness remain too theoretical in general. Motivated by this remark, in this article we revisit and extend this study to related problems, by exploiting holonomicity. In this framework, a first generalization of [14] is to recover the coefficients of both $g$ and $p$ in the exponential-polynomial case:

Problem 1 (Exp-Poly Inverse Problem). Let a measure $\mu_{f}=f \mathbb{1}_{G} d x$, supported on a compact semi-algebraic set $G$, whose algebraic boundary is included in the zero set of a polynomial $g \in \mathbb{R}[x]_{d}$. Let $f=\exp (p)$, with $p \in \mathbb{R}[x]_{s}$. Given $s, d$, and a finite number of moments $m_{\alpha},|\alpha| \leqslant N$, recover the coefficients of both $g$ and $p$.

More general, the inverse problem for holonomic weights is:

Problem 2 (General Inverse Problem). Let a measure $\mu_{f}=f \mathbb{1}_{G} d x$, supported on a compact semi-algebraic set $G$, with holonomic $f$. Given a finite number of moments $m_{\alpha},|\alpha| \leqslant N$, recover a polynomial $g \in \mathbb{R}[x]$ vanishing on the algebraic boundary of $G$ and the coefficients of a holonomic system satisfied by $f$.

Finally, we note the closely related direct problem:

Problem 3 (General Direct Problem). Let a measure $\mu_{f}=f \mathbb{1}_{G} d x$, supported on a compact semi-algebraic set, with given holonomic $f$. Find a holonomic system of recurrences for the sequence of moments $\left(m_{\alpha}\right)$.

Contributions: We address the above problems in the framework of holonomic distributions, employing well-known algorithmic properties of noncommutative polynomial representation of linear differential operators (see Section 2), as well as a generalized Stokes formula [14]. Firstly, this allows us to solve Problem 1 in Section 4.1 w we prove that this reconstruction problem boils down to solving a linear system of $3 d+s-1$ equations, involving moments up to degree $|\alpha| \leqslant 4 d+2(s-1)$.

Secondly, as a by-product, an alternate method to creative telescoping is proposed for computing linear recurrences for the moments in Section 3. The advantage is that when the coefficients of $g$ are given as parameters, the obtained recurrences stay linear with respect to them. However, there is no guarantee that this method provides a holonomic ideal. We could only prove 
that it solves Problem 3 (i.e. it provides a holonomic ideal) in the restricted case of exponential-polynomial density and $g$ nonsingular in $\mathbb{C}^{n}$.

Finally, Problem 2 is solved in Section 4.2 we prove that a holonomic system for $f$ can be found by solving a finite system of linear equations, but their number cannot be a priori bounded. Once the density is known, the support is reconstructed as solution of a similar linear system, but in this case we provide an explicit uniform bound on the number of required moments.

\section{$1.1 \quad$ Related works}

Moment problem: Concerning the moment problem, let $\mu$ be a Borel measure on $\mathbb{R}^{n}$ with all its moments finite. When $\mu$ is atomic with finitely many atoms (i.e., when $\mu=\sum_{k=1}^{d} \gamma_{k} \delta_{\xi_{k}}$, where $\delta_{\xi_{k}}$ is the Dirac measure, for some $\left(\xi_{k}\right) \subset \mathbb{R}^{n}$ and some positive weight $\left(\gamma_{k}\right)$ ), a first classical problem is to retrieve the atoms and the weights of $\mu$ from some finite truncation of its moment vector $\left(m_{\alpha}\right)_{\alpha \in \mathbb{R}^{n}}$. In [15] a thorough overview of algebraic methods for this problem is given. An important idea consists in computing a sparse polynomial-exponential representation of a multivariate series from its truncated Taylor series, whose coefficients correspond to moments. For instance, for pairwise distinct $\xi_{1}, \ldots, \xi_{d}$, the moment generating series is:

$$
\sigma_{\delta_{\xi}}(y)=\sum m_{\alpha} \frac{y^{\alpha}}{\alpha !}=\sum_{k=1}^{d} \gamma_{k} \exp \left(\xi_{k} y\right)
$$

Such generating functions are also the solutions of systems of partial differential equations with constant coefficients. Hence, the sparse representation of the polynomial-exponential (also known as Prony method) is related to the inverse system of the isolated points of the characteristic variety of this system. Methods to obtain such representation are given in [15]. Also, flat extension criteria, like for instance [12, Theorem 3.7], provide purely algebraic methods to reconstruct both the number of atoms, their values and weights function of the rank of the moment matrix.

All in all, moments of atomic measures satisfy multi-index linear recurrences with constant coefficients [15], which provide another incentive to consider the more general holonomic case. In this sense, these recurrences can be computed by creative telescoping.

Creative telescoping: These methods perform integration of functions (with free parameters), in the framework of non-commutative polynomial representation of linear differential operators (see [3, 10, 2] and references therein). In particular, the direct Problem 3 can be solved for instance by the algorithms of Oaku [17]. Based on the D-module theory (see also [5, 19]), one 
computes a holonomic system for the definite integral of a holonomic function with parameters over a domain defined by polynomial inequalities. In the algorithms, holonomic distributions are involved, so, a subtle distinction has to be made between the ideal of operators with polynomial coefficients, which correspond to holonomicity, and those with rational coefficients which correspond to so-called D-finiteness. We will come back to this in Section 2.

Also, the Lagrange Identity [8] (see also eq. (12) and Prop. 2), related to integration by parts, will play an important role in our approach. In the one variable case, for a linear differential operator with polynomial coefficients, $L=c_{r} \partial_{x}^{r}+\ldots+c_{0}$, its adjoint is defined as $L^{*}=(-1)^{r} \partial_{x}^{r} c_{r}+\ldots+c_{0}$ and the following holds:

$$
\varphi L(f)-L^{*}(\varphi) f=\partial_{x}\left(\mathcal{L}_{L}(f, \varphi)\right)
$$

for any function $\varphi$ and $f$, with an explicit $\mathcal{L}_{L}$.

Inverse problem in the univariate case: In [1], the inverse Problem 2 is solved in the univariate case, for piecewise D-finite densities. Specifically, $\mu_{f}=\sum_{i=1}^{d-1} \mathbb{1}_{\left[\xi_{i}, \xi_{i+1}\right]} f_{i} \mathrm{~d} x$, for a set of $d$ unknown points, $a=\xi_{1}<\ldots<\xi_{d}=b$, with $[a, b] \subset \mathbb{R}$, and unknown smooth D-finite functions $f_{i}$. An important observation [1, Thm 2.12] is that the associated distribution $\sum_{i=1}^{d-1} \mathbb{1}_{\left[\xi_{i}, \xi_{i+1}\right]} f_{i}$ is annihilated by some holonomic operator $\hat{L}=g(x)^{r} L$, where $g(x)=\prod_{i=1}^{d}\left(x-\xi_{i}\right)$ and the operator $L$ of order $r$ satisfies $L f_{i}=0$.

Remark 1. As noted in [1], for general holonomic operators $L$ with $r>1$, the number $N$ of required moments, in order to correctly recover the parameters, might depend also on specific coefficients of L. An example is the nth Legendre polynomial, whose first $n$ moments (taken over $[-1,1]$ ) vanish, while $L_{n}=\partial_{x}\left(\left(1-x^{2}\right) \partial_{x}\right)+n(n+1)$, hence the reconstruction of $\mu_{f}$ depends also on the parameter $n$, which enters the definition of $L_{n}$. On the contrary, for exponential-polynomial case, we show that $N$ depends only on the degrees of the polynomials involved.

As discussed above, in the univariate case, the above problems are well tackled in literature, so this article deals with the multivariate case. However, to illustrate the basic ideas, we give two elementary univariate examples of our approach, omitting the technical proofs. 


\subsection{Introductory examples}

Example 1 (Direct problem for erf-like function). We are interested in computing a recurrence for the moments $m_{i}=\int_{-1}^{1} x^{i} e^{-x^{2}} \mathrm{~d} x$. The idea is to include $\mathbb{1}_{[-1,1]}$ in the integral, and consider the distribution $u$ corresponding to $\mathbb{1}_{[-1,1]}(x) e^{-x^{2}}$. Although not differentiable as a function, u satisfies (see Sec. 2.2 for details):

$$
\left(1-x^{2}\right)\left(\partial_{x}+2 x\right) u=0 .
$$

Integrating for the test function $x^{i}$, using (3) and noticing that its right hand side vanishes after integration, one has:

$$
\int_{-1}^{1} e^{-x^{2}}\left(\partial_{x}+2 x\right)^{*}\left(\left(1-x^{2}\right) x^{i}\right)=0,
$$

which directly provides the recurrence

$$
i m_{i-1}-(i+4) m_{i+1}+2 m_{i+3}=0 .
$$

The extension of this method to the multivariate case is given in Sec. 3 .

Example 2 (Univariate support and density reconstruction). Consider the problem of reconstructing the parameters $\xi_{1}, \xi_{2}$ and $p_{2}, p_{1}, p_{0}$ such that, the first $N$ moments $\left\{m_{i}, 0 \leqslant i \leqslant N\right\}$ are known:

$$
m_{i}=\int_{\xi_{1}}^{\xi_{2}} x^{i} e^{p_{2} x^{2}+p_{1} x+p_{0}} \mathrm{~d} x .
$$

Like in the previous example, $u=\mathbb{1}_{\left[\xi_{1}, \xi_{2}\right]} e^{p_{2} x^{2}+p_{1} x+p_{0}}$ satisfies:

$$
\left(x-\xi_{1}\right)\left(x-\xi_{2}\right)\left(\partial_{x}-2 p_{2} x-p_{1}\right) u=0 .
$$

Denote by $\hat{L}:=g(x) \partial_{x}+h(x)$ the operator to be reconstructed such that $\hat{L} f=0$, with $g(x)=x^{2}+g_{1} x+g_{0}$ and $h(x)=\sum_{i=0}^{3} h_{i} x^{i}$. Integrating and using Lagrange identity, one has:

$$
\left.\int_{-\infty}^{\infty}\left(g(x) \partial_{x}-h(x)\right)\left(x^{i}\right)\right) u d x=0 .
$$


This gives for each $i \geqslant 0$ :

$i m_{i+1}+i g_{1} m_{i}+i g_{0} m_{i-1}-h_{3} m_{i+3}-h_{2} m_{i+2}-h_{1} m_{i+1}-h_{0} m_{i}=0$.

Hence, the coefficients of $g$ and $h$ are solution of the above infinite linear system. If $g$ is recovered, $p$ (except for $p_{0}$ coefficient) could also be recovered from the division $h / g$. Finally the constant coefficient $p_{0}$ could also be recovered from the equation (4), with $i=0$.

The main question is whether a truncated system (6), which considers only moments up to degree $N$, can provide the correct solution for $g$ and $h$. We will address this in Section 4. Specifically, in Thm. 11 we prove a sufficient bound for the case of an n-variable exponential-polynomial density, together with Algorithm 2 which reconstructs the coefficients. It needs in our case the first $N=10$ moments.

\section{Holonomicity and distributions}

For completeness, we start by providing a short reminder on D-finiteness versus holonomicity. Unlike more classical settings like analytic functions, the distinction between these two very related notions is essential when considering distributions. We refer to [3, 10, 17] for a more comprehensive presentation.

\subsection{Differential operators and holonomicity}

Consider the following rings of linear partial differential operators:

(i) The ring of differential operators with polynomial coefficients (the $n$-th Weyl algebra) $\mathfrak{D}_{n}:=\mathbb{K}\left[x_{1}, \ldots, x_{n}\right]\left\langle\partial_{x_{1}}, \ldots, \partial_{x_{n}}\right\rangle$, generated by $\left\{x_{1}, \ldots, x_{n}, \partial_{x_{1}}, \ldots, \partial_{x_{n}}\right\}$ and quotiented by the relations:

$$
\partial_{x_{i}} x_{j}=\left\{\begin{array}{ll}
x_{i} \partial_{x_{i}}+1, & i=j, \\
x_{j} \partial_{x_{i}}, & i \neq j,
\end{array} \quad x_{i} x_{j}=x_{j} x_{i}, \quad \partial_{x_{i}} \partial_{x_{j}}=\partial_{x_{j}} \partial_{x_{i}} .\right.
$$

We have that $\left\{x^{\beta} \partial_{x}^{\alpha}, \alpha, \beta \in \mathbb{N}^{n}\right\}$ is a basis of $\mathfrak{D}_{n}$ as a $\mathbb{K}$-vector space. If $L=\sum_{\alpha, \beta} c_{\alpha, \beta} x^{\beta} \partial_{x}^{\alpha}$, its order is the largest value of $|\alpha|$ such that there exists $\beta$ with $c_{\alpha, \beta} \neq 0$.

(ii) The ring of differential operators with rational fraction coefficients (Ore Algebra) $\mathfrak{D}_{n}^{*}:=\mathbb{K}\left(x_{1}, \ldots, x_{n}\right)\left\langle\partial_{1}, \ldots, \partial_{n}\right\rangle$, where the commutation rules of $\mathfrak{D}_{n}$ are extended by

$$
\partial_{x_{i}} q(x)=q(x) \partial_{x_{i}}+\frac{\partial q(x)}{\partial x_{i}}, \quad q(x) \in \mathbb{K}\left(x_{1}, \ldots, x_{n}\right) .
$$


Differential operators in $\mathfrak{D}_{n}$ naturally act on smooth functions via $\partial_{x_{i}} f=$ $f_{x_{i}}:=\frac{\partial f}{\partial x_{i}}$. The annihilator $\mathfrak{A} \mathfrak{n} \mathfrak{n}(f)$ is a left ideal of $\mathfrak{D}_{n}$ :

$$
\mathfrak{A} \mathfrak{n} \mathfrak{n}(f):=\left\{L \in \mathfrak{D}_{n} \mid L f=0\right\} .
$$

One can also see $\mathfrak{A} \mathfrak{n} \mathfrak{n}(f)$ as a left ideal of $\mathfrak{D}_{n}^{*}$, and the quotient $\mathfrak{D}_{n}^{*} / \mathfrak{A} \mathfrak{n} \mathfrak{n}(f)$ as a $\mathbb{C}\left(x_{1}, \ldots, x_{n}\right)$-vector space. A smooth function $f$ is called $D$-finite if $\mathfrak{D}_{n}^{*} / \mathfrak{A} \mathfrak{n} \mathfrak{n}(f)$ has finite dimension. Equivalently, its iterated derivatives $\left\{\partial_{x}^{\alpha} f, \alpha \in \mathbb{N}^{n}\right\}$ form a finite-dimensional vector space over rational fractions.

In other cases, when $f$ is a "generalized function", for instance a distribution, $\mathfrak{A} \mathfrak{n} \mathfrak{n}(f)$ can only be seen as a left ideal of $\mathfrak{D}_{n}$ and $\mathfrak{D}_{n} / \mathfrak{A} \mathfrak{n} \mathfrak{n}(f)$ as a $\mathbb{C}$-vector space. For example, the univariate Dirac distribution, defined by $\langle\delta, f\rangle=f(0)$, is annihilated (as a distribution) by $x$, since $\langle x \delta, f\rangle=$ $\langle\delta, x f\rangle=0$. However, a left ideal of $\mathfrak{D}_{1}^{*}$ containing $x$ is necessarily $\mathfrak{D}_{1}^{*}$, but 1 annihilating $\delta$ would imply $\delta=0$. In that setting, the relevant notion is holonomicity.

Definition 1. Let $\mathfrak{I}$ be a left ideal of $\mathfrak{D}_{n}$. For $L \in \mathfrak{D}_{n}$, let $[L]_{\mathfrak{I}}$ denote the class of $L$ in the quotient $\mathfrak{D}_{n} / \mathfrak{I}$. For $s \geqslant 0$, define

$$
\left(\mathfrak{D}_{n} / \mathfrak{I}\right)_{s}=\operatorname{Span}_{\mathbb{K}}\left\{\left[x^{\beta} \partial_{x}^{\alpha}\right]_{\mathfrak{I}},|\alpha|+|\beta| \leqslant s\right\} .
$$

Then there exists a polynomial $b(s) \in \mathbb{K}[s]$ such that $\operatorname{dim}_{\mathbb{K}}\left(\mathfrak{D}_{n} / \mathfrak{I}\right)_{s}=b(s)$ for $s$ large enough. The degree of $b(s)$ is called the Bernstein dimension of $\mathfrak{D}_{n} / \mathfrak{I}$. The left ideal $\mathfrak{I}$ is called holonomic if the Bernstein dimension of $\mathfrak{D}_{n} / \mathfrak{I}$ is equal to $n$.

The well-known notion of Gröbner bases was generalized to this noncommutative setting (see for example [5, 3, 10] and references therein). This is the building block of efficient closure operations for D-finite [3, 10] or holonomic [5, 19, 17] objects, thus allowing for their algorithmic treatment.

Similarly, $\mathfrak{R}_{n}:=\mathbb{K}\left[\alpha_{1}, \ldots, \alpha_{n}\right]\left\langle S_{\alpha_{1}}, \ldots, S_{\alpha_{n}}\right\rangle$ is the set of difference operators with polynomial coefficients in $\alpha$, acting on sequences $u=\left(u\left(\gamma_{1}, \ldots, \gamma_{n}\right)\right)_{\gamma \in \mathbb{N}^{n}}$ via

$$
\begin{aligned}
\left(\alpha_{i} u\right)\left(\gamma_{1}, \ldots, \gamma_{n}\right) & =\gamma_{i} u\left(\gamma_{1}, \ldots, \gamma_{n}\right), & \text { and } \\
\left(S_{\alpha_{i}} u\right)\left(\gamma_{1}, \ldots, \gamma_{n}\right) & =u\left(\gamma_{1}, \ldots, \gamma_{i}+1, \ldots, \gamma_{n}\right), & \gamma \in \mathbb{N}^{n}
\end{aligned}
$$

The annihilator $\mathfrak{A} \mathfrak{n} \mathfrak{n}(u)=\left\{R \in \mathfrak{R}_{n} \mid R u=0\right\}$ is the set of recurrence relations satisfied by $u$, which is holonomic when its generating series is holonomic [3]. 


\subsection{Holonomic distributions}

Introduced by Schwartz [18], distributions generalize functions and measures. A minimal introduction to this topic is provided below.

Definition 2 (Test functions and distributions). Let $\mathcal{E}=\mathcal{C}^{\infty}\left(\mathbb{R}^{n}\right)$ be the set of smooth functions over $\mathbb{R}^{n}$, equipped with the compact-open topology: $\varphi_{k} \rightarrow \varphi$ in $\mathcal{E}$ if $\partial_{x}^{\alpha} \varphi_{k}$ converges uniformly to $\partial_{x}^{\alpha} \varphi$ over every compact set, for each $\alpha \in \mathbb{N}^{n}$.

Its topological dual $\mathcal{E}^{\prime}$ is the set of compactly supported distributions (or simply distributions in this article) i.e. linear forms $T: \mathcal{E} \rightarrow \mathbb{R}$ such that:

- There exists a minimal compact set $K \subseteq \mathbb{R}^{n}$ (the support of $T$ ) such that $\langle T, \varphi\rangle=0$ whenever $\varphi$ vanishes over $K$.

- $\left\langle T, \varphi_{k}\right\rangle \rightarrow 0$ whenever $\varphi_{k} \rightarrow 0$ in $\mathcal{E}$.

$\mathcal{E}^{\prime}$ has a canonical $\mathfrak{D}_{n}$-module structure:

$$
\langle L T, \varphi\rangle:=\left\langle T, L^{*} \varphi\right\rangle, \quad L \in \mathfrak{D}_{n}, T \in \mathcal{E}^{\prime}, \varphi \in \mathcal{E},
$$

where the adjoint operator $L^{*}$ is defined by

$$
x_{i}^{*}=x_{i}, \quad \partial_{x_{i}}^{*}=-\partial_{x_{i}}, \quad \text { and } \quad\left(L_{1} L_{2}\right)^{*}=L_{2}^{*} L_{1}^{*} .
$$

Definition 3 (Holonomic distribution). A distribution $T \in \mathcal{E}^{\prime}$ is holonomic if its annihilator is a holonomic ideal of $\mathfrak{D}_{n}$ :

$$
\mathfrak{A} \mathfrak{n}(T):=\left\{L \in \mathfrak{D}_{n} \mid L T=0 \text { as a distribution }\right\} .
$$

A measure supported on a set $G$, with density $f \in \mathcal{E}$, is represented by the distribution $f \mathbb{1}_{G}$, with $\left\langle f \mathbb{1}_{G}, \varphi\right\rangle=\int_{G} \varphi(x) f(x) \mathrm{d} x$.

We make the following assumption on $G \subseteq \mathbb{R}^{n}$ :

Assumption 1. $G$ is a compact n-dimensional semi-algebraic set. In particular, the following holds:

(1) $G$ is an $n$-dimensional compact manifold such that its boundary can be decomposed as $\partial G=Z \cup Z^{\prime}$, with $Z$ a finite union of $(n-1)$-dimensional manifolds and $Z^{\prime}$ a negligible set w.r.t the $(n-1)$-dimensional Hausdorff measure.

(2) the ideal of polynomials vanishing over $\partial G$ is radical and principal i.e., generated by a single square-free polynomial $g$. In particular, the family $\left\{g, g_{x_{1}}, \ldots, g_{x_{n}}\right\}$ is coprime, implying that the set of singular points $\{x \mid g(x)=0$ and $\nabla g(x)=0\}$ is negligible in $\partial G$. 


\subsection{Moments of a distribution}

Definition 4 (Moments of a compactly supported distribution). The moments of a distribution $T \in \mathcal{E}^{\prime}$ are:

$$
m_{\alpha}(T):=\left\langle T, x^{\alpha}\right\rangle, \quad \alpha \in \mathbb{N}^{n} .
$$

Note that if $T=f \mathbb{1}_{G}$ with $G$ compact and $f \in \mathcal{E}$, then $m_{\alpha}\left(f \mathbb{1}_{G}\right)$ coincides with the moments defined in eq. (1).

A convenient way to deal with moments of a distribution is the Fourier transform (also called characteristic function).

Definition 5. The Fourier transform of a distribution $T \in \mathcal{E}^{\prime}$ is the analytic function $\mathscr{F}\{T\}$ of $z=\left(z_{1}, \ldots, z_{n}\right) \in \mathbb{R}^{n}$ defined by:

$$
\mathscr{F}\{T\}(z)=\sum_{\alpha \in \mathbb{N}^{n}} m_{\alpha}(T) \frac{(-\mathbf{i} z)^{\alpha}}{\alpha !}=\left\langle T, e^{-\mathbf{i} x \cdot z}\right\rangle, \quad z \in \mathbb{R}^{n} .
$$

Proposition 1. Let $T \in \mathcal{E}^{\prime}$ and $L=\sum_{\beta} q_{\beta}(x) \partial_{x}^{\beta} \in \mathfrak{D}_{n}$.

(i) The Fourier transform of $L T$ is related to that of $T$ by

$$
\begin{aligned}
& \mathscr{F}\{L T\}=L^{\mathscr{F}} \mathscr{F}\{T\}, \text { with } \\
& L^{\mathscr{F}}=L\left[\begin{array}{cc}
x_{i} & \mapsto \mathbf{i} \partial_{z_{i}} \\
\partial_{x_{i}} \mapsto \mathbf{i} z_{i}
\end{array}\right]=\sum_{\beta} q_{\beta}\left(\mathbf{i} \partial_{z}\right)(\mathbf{i} z)^{\beta} .
\end{aligned}
$$

(ii) The moments of $L T$ are related to those of $T$ by

$$
\begin{aligned}
& \left(m_{\alpha}(L T)\right)=L^{\mathscr{M}}\left(m_{\alpha}(T)\right), \quad \text { with } \\
& L^{\mathscr{M}}=L\left[\begin{array}{ll}
x_{i} & \mapsto S_{\alpha_{i}} \\
\partial_{x_{i}} \mapsto-\alpha_{i} S_{\alpha_{i}}^{-1}
\end{array}\right]=\sum_{\beta}(-1)^{|\beta|} q_{\beta}\left(S_{\alpha}\right)\left(\prod_{i=1}^{n}\left(\alpha_{i} S_{\alpha_{i}}^{-1}\right)^{\beta_{i}}\right),
\end{aligned}
$$

Proof. Similar to [15, Sec. 5.1.] (see A.1 for completeness).

Proposition 2. Let $T \in \mathcal{E}^{\prime}$. An operator $L \in \mathfrak{D}_{n}$ satisfies

$$
\left\langle T, L^{*} x^{\alpha}\right\rangle=0, \quad \text { for all } \alpha \in \mathbb{N}^{n},
$$

if and only if $L \in \mathfrak{A} \mathfrak{n} \mathfrak{n}(T)$.

Proof. By the injectivity of the Fourier transform on compactly supported distributions [18]. 


\section{Direct problem for moments}

As mentioned in the introduction, the direct Problem 3 can be solved using an algorithm presented in [17]. However, one may ask whether the simple roadmap of Example 1 can be generalized to the multivariate case and provide a more efficient method. For that, firstly, Lagrange identity in the multivariate setting is needed:

Lemma 1 (Lagrange identity). For $f, g \in \mathcal{E}$ and $L \in \mathfrak{D}_{n}$ of order $r$, there exists a vector field $\mathcal{L}_{L}(f, g): \mathbb{R}^{n} \rightarrow \mathbb{R}^{n}$, called bilinear concomitant, depending on $L$ and linear in $f$ and $g$, such that:

$$
(L f) g-f\left(L^{*} g\right)=\nabla \cdot \mathcal{L}_{L}(f, g) .
$$

Each component of $\mathcal{L}_{L}(f, g)$ can be written

$$
\mathcal{L}_{L, i}(f, g)=\sum_{|\alpha|+|\beta| \leqslant r-1} c_{L, i, \alpha, \beta}(x)\left(\partial_{x}^{\alpha} f\right)\left(\partial_{x}^{\beta} g\right), \quad i \in[1 \ldots n] .
$$

with coefficients $c_{L, i, \alpha, \beta}(x) \in \mathbb{K}[x]$ depending on $L$.

Secondly, the action of differential operators on compactly supported distributions of the form $f \mathbb{1}_{G}$ is provided:

Proposition 3. Let $G$ as in Assumption 1 , $f \in \mathcal{E}$ and $L \in \mathfrak{D}_{n}$. Then the distribution $L\left(f \mathbb{1}_{G}\right)$ admits the following expression:

$$
\left\langle L\left(f \mathbb{1}_{G}\right), \varphi\right\rangle=\int_{G} \varphi(L f) \mathrm{d} x-\int_{\partial G} \mathcal{L}_{L}(f, \varphi) \cdot \boldsymbol{n} \mathrm{d} S
$$

where $\boldsymbol{n}$ and $\mathrm{d} S$ respectively denote the normal vector and the $(n-1)$ dimensional Hausdorff measure on $\partial G$.

Proof. Integrating Lagrange's identity 12 with $g=\varphi$ and using the divergence theorem, we have:

$$
\int_{G} \varphi(L f) \mathrm{d} x-\int_{G}\left(L^{*} \varphi\right) f \mathrm{~d} x=\int_{\partial G} \mathcal{L}_{L}(f, \varphi) \cdot \boldsymbol{n} \mathrm{d} S .
$$

Following [14], the divergence theorem is a consequence of Stokes' theorem when $\partial G$ is smooth, or of a generalization by Whitney [20, Theorem 14A] when $G$ satisfies Assumption 1.(1).

Finally, the following proposition provides differential equations for measures supported on semi-algebraic sets. 
Proposition 4. Let $G$ and $g$ as in Assumption 1 , $f \in \mathcal{E}, L \in \mathfrak{A} \mathfrak{n} \mathfrak{n}(f)$ of order $r$. Then $g^{r} L \in \mathfrak{A} \mathfrak{n} \mathfrak{n}\left(f \mathbb{1}_{G}\right)$.

Proof. Using Prop. 3, one needs to prove that $\int_{G} g^{r} \varphi(L f) \mathrm{d} x$ and $\int_{\partial G} \mathcal{L}_{L}\left(f, g^{r} \varphi\right)$. $\boldsymbol{n} \mathrm{d} S$ are zero. The first one is trivial since $L \in \mathfrak{A} \mathfrak{n} \mathfrak{n}(f)$. For the second, $\mathcal{L}_{L}\left(f, g^{r} \varphi\right)$ involves derivatives $\partial_{x}^{\alpha}\left(g^{r} \varphi\right)$ with $|\alpha|<r$ (Lemma 1), so it vanishes over $\partial G$.

Hence, Proposition 4 gives an easy way to construct operators in $\mathfrak{A} \mathfrak{n} \mathfrak{n}\left(f \mathbb{1}_{G}\right)$ from operators in $\mathfrak{A} \mathfrak{n} \mathfrak{n}(f)$. Indeed, given a Gröbner basis $\left\{L_{1}, \ldots, L_{k}\right\}$ of $\mathfrak{A n n}(f)$, and $g \in \mathbb{R}[x]$ vanishing over $\partial G$, each operator $g^{r_{i}} L_{i}$ (with $r_{i}$ the order of $L_{i}$ ) annihilates $f \mathbb{1}_{G}$ as a distribution. Therefore, each operator $R_{i}:=\left(g^{r_{i}} L_{i}\right)^{\mathscr{M}}$ gives a valid recurrence for the sequence of moments $\left(m_{\alpha}\right)$.

However, from the fact that $f$ is holonomic one can not directly guarantee that the ideal generated by $\left\{g^{r_{1}} L_{1}, \ldots, g^{r_{k}} L_{k}\right\}$ is holonomic. Similarly, we are not able to prove (or refute) that $\left\{R_{1}, \ldots, R_{k}\right\}$ is holonomic in general. Nevertheless, one can apply a Gröbner basis algorithm to it, which will possibly terminate and return such a basis. This heuristic is proposed in Algorithm 1. We prove that this algorithm terminates, in the particular case

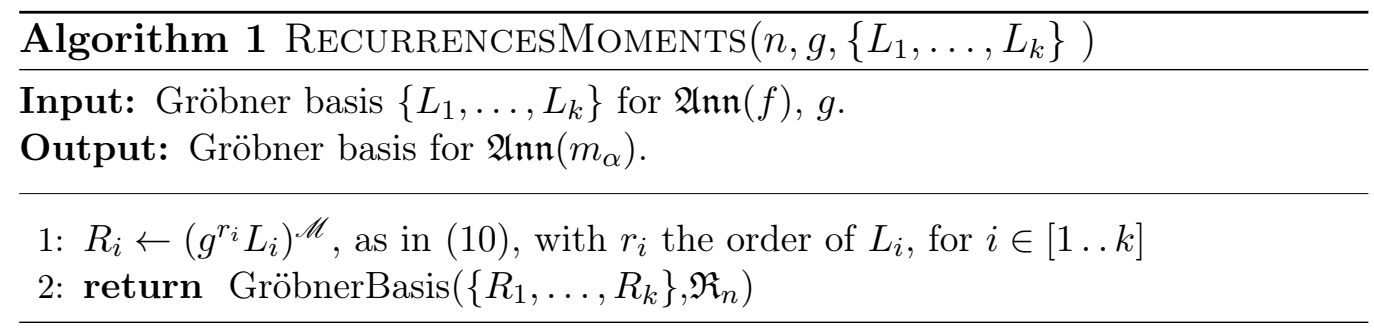

of an exponential-polynomial density (including the Lebesgue measure), and a smooth boundary, extending [17, Prop. 4].

Proposition 5. Let $f(x)=e^{p(x)}$ with $p \in \mathbb{R}[x]_{s}$, and $g \in \mathbb{R}[x]_{d}$ vanishing over $\partial G$. Suppose moreover that $g$ is nonsingular in $\mathbb{C}^{n}$, that is, there exists no $x \in \mathbb{C}^{n}$ such that $g(x)=0$ and $\nabla g(x)=0$.

(i) The operators

$$
L_{i}=g\left(\partial_{x_{i}}-p_{x_{i}}\right), \quad i \in[1 \ldots n],
$$

are generators of an holonomic ideal $\mathfrak{I}$ contained in $\mathfrak{A} \mathfrak{n} \mathfrak{n}\left(f \mathbb{1}_{G}\right)$.

(ii) The operators $L_{i}^{\mathscr{F}}(i \in[1 \ldots n])$ span a holonomic ideal $\mathfrak{I}^{\mathscr{F}}$ contained in $\mathfrak{A n n}\left(\mathscr{F}\left\{f \mathbb{1}_{G}\right\}\right)$. 
Proof. For $(i)$, first note that the operators $L_{i}$ also generate

$$
\begin{aligned}
L_{i j} & :=\left(\partial_{x_{j}}-p_{x_{j}}\right) L_{i}-\left(\partial_{x_{i}}-p_{x_{i}}\right) L_{j} \\
& =g_{x_{j}}\left(\partial_{x_{i}}-p_{x_{i}}\right)-g_{x_{i}}\left(\partial_{x_{j}}-p_{x_{j}}\right), \quad 1 \leqslant i<j \leqslant n .
\end{aligned}
$$

Holonomicity is proved via the characteristic variety, as for instance in [17]. For $L=\sum_{|\alpha| \leqslant r} q_{\alpha}(x) \partial_{x}^{\alpha}$ of order $r$, define its principal symbol $\sigma(L)(x, \xi)=$ $\sum_{|\alpha|=r} q_{\alpha}(x) \xi^{\alpha}$ for $(x, \xi) \in \mathbb{C}^{2 n}$. Then for a left ideal $\mathfrak{I}, \operatorname{Char}(\mathfrak{I})=\{(x, \xi) \in$ $\left.\mathbb{C}^{2 n} \mid \sigma(L)(x, \xi)=0 \forall L \in \mathfrak{I} \backslash\{0\}\right\}$. With these notations, $\mathfrak{I}$ is holonomic if and only if all the components of $\operatorname{Char}(\mathfrak{I})$ are of dimension at most $n$. In our case,

$$
\sigma\left(L_{i}\right)(x, \xi)=g(x) \xi_{i}, \quad \text { and } \quad \sigma\left(L_{i j}\right)(x, \xi)=g_{x_{j}}(x) \xi_{i}-g_{x_{i}}(x) \xi_{j} .
$$

Hence, if $(x, \xi) \in \operatorname{Char}(\mathfrak{I})$, then either $g(x) \neq 0$, implying $\xi=0$, or $g(x)=0$. In the latter case, $\nabla g(x) \neq 0$ (since $g$ is nonsingular) and hence there exists $\lambda \in \mathbb{C}$ s.t. $\xi=\lambda \nabla g(x)$. In both cases, the corresponding components of Char $(\mathfrak{I})$ have dimension $n$.

For $(i i)$, since the Fourier transform maps $x_{i}$ to $\mathbf{i} \partial_{z_{i}}$ and $\partial_{x_{i}}$ to $\mathbf{i} z_{i}$, it is clear that $\mathfrak{I}$ is holonomic if and only if $\mathfrak{I}^{\mathscr{F}}$ is holonomic.

Interesting enough, for the examples we tried for an exponential-polynomial density, Algorithm 1 always terminated, even when the boundary was not smooth (see Example 3). Also, it was faster that "classical" creative telescoping, which firstly constructs a Gröbner basis for $f \mathbb{1}_{G}$ and then applies Takayama algorithm [17]. Further investigation is needed to provide a comparison in this case.

However, having a Gröbner basis is not mandatory for the reconstruction problem addressed in the next section. The recurrences obtained as above turn out to be sufficient and constitute the basic brick of our reconstruction method.

\section{Reconstruction methods}

Given some moments $m_{\alpha}\left(f \mathbb{1}_{G}\right)$ associated to a measure of unknown D-finite density $f \in \mathcal{E}$ and unknown compact algebraic support $G$, our goal is to reconstruct a polynomial $\tilde{g}$ vanishing on the boundary $\partial G$ of $G$ and operators $\tilde{L} \in \mathfrak{A} \mathfrak{n} \mathfrak{n}(f)$.

The general approach is the following: 
- Take an ansatz $L^{\prime}=\sum_{\beta \in A} q_{\beta}(x) \partial_{x}^{\beta}$, for a specified finite set $A \subset \mathbb{N}^{n}$ and polynomials $q_{\beta}(x)$ with specified degrees $d_{\beta}$.

- Let $R=L^{\prime \mathscr{M}}$. Solve a finite-dimensional linear system in the unknown coefficients of the polynomials $q_{\beta}$ :

$$
\left(R m\left(f \mathbb{1}_{G}\right)\right)_{\alpha}=0, \quad|\alpha| \leqslant N .
$$

This requires the knowledge of moments $m_{\alpha}\left(f \mathbb{1}_{G}\right)$ with $|\alpha| \leqslant N+$ $\max _{\beta \in A}\left\{d_{\beta}-|\beta|\right\}$ (see eq. (10)).

- From the solution $L^{\prime}$ of (17), extract a polynomial $g$ vanishing on $\partial G$ and an operator $\tilde{L} \in \mathfrak{A} \mathfrak{n} \mathfrak{n}(f)$.

Note that the solution of 17 corresponds to a truncation of the infinite system (11), since $\left\langle f \mathbb{1}_{G}, L^{*} x^{\alpha}\right\rangle=0$, for $|\alpha| \leqslant N$. Hence one is interested in obtaining bounds $\hat{N}$ on $N$, such that any solution of (17) is also solution of (11). Such an a priori uniform bound depending only on $A$ and $d_{\beta}$ does not exist in general, cf. Remark 1

Another issue is that $L^{\prime}$ may not be factorized as $\tilde{g}(x)^{r} \tilde{L}$ with $\tilde{g}$ vanishing on $\partial G$ and $\tilde{L} f=0$. See for instance the operator in 16 .

In Section 4.1, we solve both issues when $f$ is exponential-polynomial and give the associated algorithm. Then, in Section 4.2, we address the general holonomic case in two steps: firstly, for recovering the density, we prove that $N$ is finite, but no a priori bound for it is known; secondly, once the density is known, a stronger result is proved for the support reconstruction, since an explicit uniform bound on the number of required moments is given.

In the algorithms proposed below, "exact computations" are assumed, that is, both the polynomial coefficients and the given moments $m_{\alpha}$ lie in a computable finite extension of $\mathbb{Q}$. The practical case of approximately known numerical moments is briefly analyzed in Section 5 .

\subsection{Exponential-polynomial densities}

Let $f(x)=\exp (p(x))$ with $\operatorname{deg} p=s$, together with $G$ and $g$ as in Assumption 1. $\operatorname{deg} g=d$. Then $f$ is annihilated by $L_{i}=\partial_{x_{i}}-p_{x_{i}}$ for $i \in[1 \ldots n]$. Algorithm 2 follows the general approach above, with ansatz $L_{i}^{\prime}=h_{0} \partial_{x_{i}}-h_{i}$ $(i \in[1 \ldots n])$ for unknown polynomials $h_{0}, \ldots, h_{n}$ where $\operatorname{deg} h_{0} \leqslant d$ and $\operatorname{deg} h_{i} \leqslant d+s-1$ for $i \in[1 \ldots n]$. Theorem 1 establishes its correctness, with an explicit bound $\hat{N}$. 


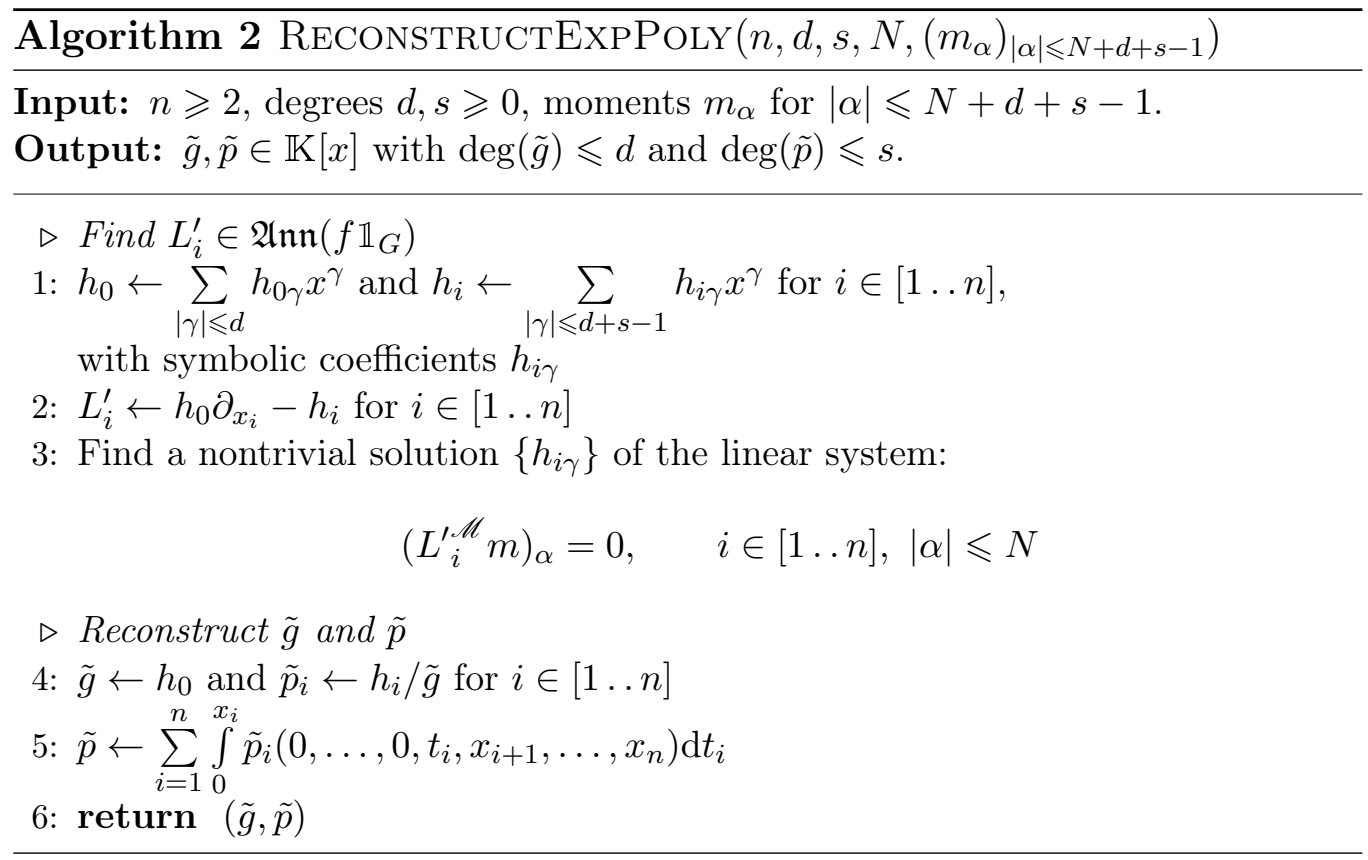

Theorem 1. Let $f(x)=\exp (p(x))$ with $\operatorname{deg} p=s$, and $G, g$ with $\operatorname{deg} g=d$ as in Assumption 1 . If $N \geqslant \hat{N}=3 d+s-1$, then ReCONSTRUCTEXP$\operatorname{Poly}\left(n, d, s, N,\left(m_{\alpha}\right)\right)$ returns $\tilde{g}=\lambda g$ with $\lambda \in \mathbb{K}^{*}$, and $\tilde{p}=p-p(0)$. This requires moments up to degree $4 d+2(s-1)$.

Moreover, if $g \geqslant 0$ over $G, \hat{N}$ can be only $2 d+s-1$, requiring moments up to degree $3 d+2(s-1)$.

Remark 2. This method cannot reconstruct the constant coefficient of $p$, which is the scaling factor of the density. In case of a probability measure over $\mathbb{R}^{n}$, this coefficient is uniquely recovered by imposing $\int_{\mathbb{R}^{n}} \exp (\tilde{p}(x)) \mathrm{d} x=1$. Otherwise, one can compute $p(0)=\log \left(m_{0} / \int_{G} \exp (\tilde{p}(x)) \mathrm{d} x\right)$, for example.

Proof. First, $\left\{h_{0} \leftarrow g, h_{i} \leftarrow g p_{x_{i}}, i \in[1 \ldots n]\right\}$ is a solution of the linear system in line 3 . Hence, one can always get a solution with $h_{0} \neq 0$. Then $\left\langle L_{i}^{\prime}\left(f \mathbb{1}_{G}\right), \varphi\right\rangle=0$ for all $i \in[1 \ldots n]$ and $\varphi \in \mathbb{K}[x]_{N}$. Using Proposition 3 , this expands to:

$$
\int_{G}\left(h_{0} p_{x_{i}}-h_{i}\right) \varphi f \mathrm{~d} x+\int_{\partial G} h_{0} \varphi f \boldsymbol{e}_{\boldsymbol{i}} \cdot \boldsymbol{n} \mathrm{d} S=0 .
$$

With $\varphi=\left(h_{0} p_{x_{i}}-h_{i}\right) g^{2}$ of degree at most $d+(s-1)+2 d \leqslant N$, the second integral is zero since $g$ vanishes over $\partial G$. Hence the first integral is zero too. Therefore, its integrand $\left(h_{0} p_{x_{i}}-h_{i}\right)^{2} g^{2}$ is zero almost everywhere over $G$. Since $G$ has nonempty interior and $f>0, g \neq 0$, this necessarily implies $h_{i}=h_{0} p_{x_{i}}$ for all $i \in[1 \ldots n]$. 
Now, the first integral in (18) being always zero for all polynomial $\varphi$ with $\operatorname{deg} \varphi \leqslant N$, so is the second. Noticing that $\boldsymbol{e}_{\boldsymbol{i}} \cdot \boldsymbol{n}=g_{x_{i}} /\|\nabla g\|$ when $\nabla g \neq 0$, and by taking $\varphi=h_{0} g_{x_{i}}$ of degree at most $2 d-1 \leqslant N$, we have

$$
\int_{\partial G}\left(h_{0} p_{x_{i}}\right)^{2} \frac{f}{\|\nabla g\|} \mathrm{d} S=0 .
$$

By summing this equality for $i \in[1 \ldots n]$, we get that $h_{0}\|\nabla g\|$ vanishes over $\partial G$. Since by Assumption 1. (2), $\{x \in \partial G \mid \nabla g(x)=0\}$ is negligible in $\partial G$, we have that $h_{0}$ (of degree at most $d$ ) vanishes over $\partial G$, whence $h_{0}=\lambda g$ since $g$ is square-free.

Finally, $\tilde{p}=p-p(0)$ is reconstructed from $p_{x_{i}}=\tilde{p}_{i}$ in line 5 .

For the case where $g \geqslant 0$ over $\partial G$, the first step of the proof still holds with $\varphi=\left(h_{0} p_{x_{i}}-h_{i}\right) g$, of degree $2 d+s-1$, in (18).

\subsection{Holonomic densities}

For higher order holonomic operators, the proof of Thm. 1 cannot be generalized: the key argument for deducing a uniform bound $\hat{N}$ was to write in (18), $\int_{G} \varphi\left(L^{\prime} f\right) \mathrm{d} x$ as $\int_{G} h \varphi f \mathrm{~d} x$, with $h \in \mathbb{K}[x]$.

Instead, we proceed in two steps. Firstly in Section 4.2.1, a holonomic system for $f$ is reconstructed, but it requires a finite number $N$ of linear equations, which cannot be a priori bounded. Secondly, the support is reconstructed Section 4.2.2.

\subsubsection{Reconstructing the density}

Algorithm 3 produces a holonomic ideal $\mathfrak{I} \subseteq \mathfrak{A} \mathfrak{n} \mathfrak{n}(f)$ spanned by a rectangular system $\left\{L_{1}, \ldots, L_{n}\right\}$, that is $L_{i} \in \mathfrak{A} \mathfrak{n} \mathfrak{n}(f) \cap \mathbb{K}[x]\left\langle\partial_{x_{i}}\right\rangle$ only involves derivatives w.r.t $x_{i}$. For that, it is sufficient to find operators annihilating $f \mathbb{1}_{G}$.

Proposition 6. Let $f$ analytic over $G$ satisfying Assumption 1 . Then $\mathfrak{A n n}\left(f \mathbb{1}_{G}\right) \subseteq$ $\mathfrak{A} \mathfrak{n} \mathfrak{n}(f)$.

Proof. Let $L^{\prime} \in \mathfrak{A} \mathfrak{n n}\left(f \mathbb{1}_{G}\right)$ of order $r$. Prop. 3 with $\varphi=g^{2 r}\left(L^{\prime} f\right)$ gives:

$$
\int_{G} g^{2 r}\left(L^{\prime} f\right)^{2} \mathrm{~d} x=0 .
$$

This implies that the analytic function $g^{r}\left(L^{\prime} f\right)$ vanishes over $G$ of nonempty interior, hence is 0 . Since $g \neq 0, L^{\prime} f=0$.

Theorem 2 guarantees that Algorithm 3 always returns an $L \in \mathfrak{A} \mathfrak{n} \mathfrak{n}(f)$ for $N$ large enough. 


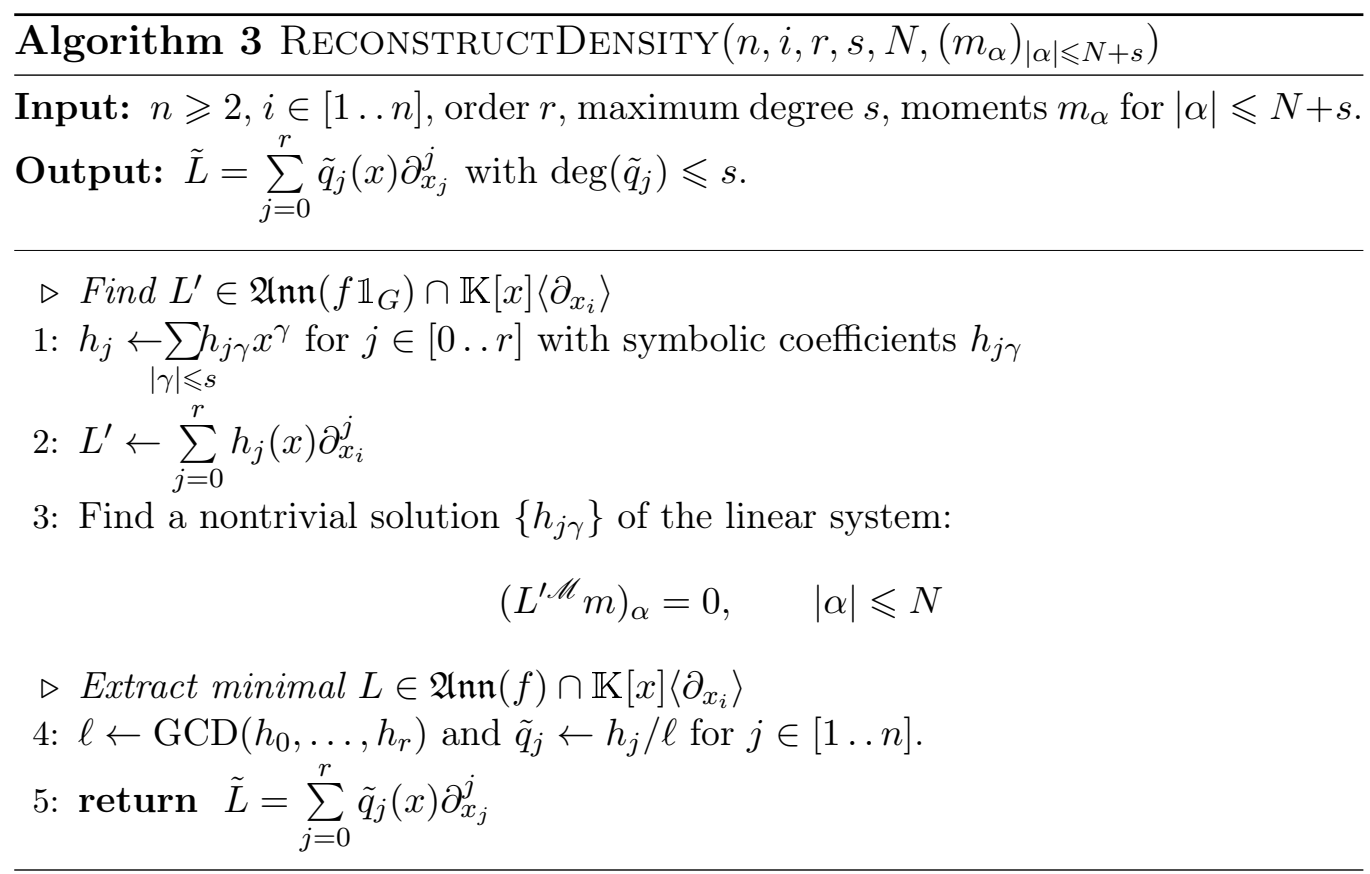

Theorem 2. Let $i \in[1 \ldots n], f$ analytic, $G, g \in \mathbb{K}[x]_{d}$ satisfying Assumption 1, and $L=\sum_{j=0}^{r} q_{j}(x) \partial_{x_{i}}^{j} \in \mathfrak{A} \mathfrak{n} \mathfrak{n}(f) \cap \mathbb{K}[x]\left\langle\partial_{x_{i}}\right\rangle$ of minimal order $r$, with $q_{r}$ of minimal degree. Then, Algorithm ReconstructDensity $\left(n, i, r, s, N,\left(m_{\alpha}\right)\right)$ returns $\tilde{L}=\lambda L$ with $\lambda \in \mathbb{K}^{*}$ for $s \geqslant d r+\max \left\{\operatorname{deg}\left(q_{j}\right)\right\}$ and $N$ large enough.

Proof. The linear system line 3 always has $g^{r} L$ as solution, by Proposition 4 . Now let $K_{N}$ denote the kernel of this system, that is $L^{\prime} \in K_{N}$ iff $\left\langle L^{\prime}\left(f \mathbb{1}_{G}\right), x^{\alpha}\right\rangle$ for all $|\alpha| \leqslant N$. The infinite inclusion chain of finite-dimensional linear subspaces $\cdots \subseteq K_{N} \subseteq K_{N+1} \subseteq \ldots$ is necessarily stationary. So for $N$ large enough, $L^{\prime} \in K_{N}$ implies $\left\langle L^{\prime}\left(f \mathbb{1}_{G}\right), x^{\alpha}\right\rangle=0$ for all $\alpha$ and hence $L^{\prime} \in$ $\mathfrak{A} \mathfrak{n} \mathfrak{n}\left(f \mathbb{1}_{G}\right)$ by Proposition 2. Finally, $L^{\prime} \in \mathfrak{A} \mathfrak{n} \mathfrak{n}(f)$ by Proposition 6

The coefficients $\left\{\tilde{q}_{0}, \ldots, \tilde{q}_{r}\right\}$ of the returned operator $\tilde{L}=\sum \tilde{q}_{j} \partial_{x_{i}}^{j}$ form a coprime family (line 4 ). This is also true for $\left\{q_{0}, \ldots, q_{r}\right\}$ by minimality of $\operatorname{deg}\left(q_{r}\right)$. By minimality of $r$, we have $\tilde{q}_{r} L-q_{r} \tilde{L}=0$, that is $\tilde{q}_{r} q_{j}=q_{r} \tilde{q}_{j}$ for all $j$. Since $\mathbb{K}[x]$ has the unique factorization property, there exists $\lambda \in \mathbb{K}$ s.t. $\tilde{q}_{r}=\lambda q_{r}$, yielding $\tilde{L}=\lambda L$. 


\subsubsection{Reconstructing the support}

From now on, we assume that a rectangular system $\left\{L_{1}, \ldots, L_{n}\right\}$ for the density $f$ is known, and that $L_{i}$ have same order $r$ L $^{1}$ Let:

$$
L_{i}=\sum_{j=0}^{r} q_{i, j}(x) \partial_{x_{i}}^{j} \in \mathfrak{A} \mathfrak{n n}(f) \cap \mathbb{K}[x]\left\langle\partial_{x_{i}}\right\rangle, \quad i \in[1 \ldots n] .
$$

The next assumption is crucial for support reconstruction. Roughly speaking, the differential system must not be singular over the Zariski closure of $\partial G$, except for a zero-measure set.

Assumption 2. The pair $\left\{g, q_{i, r}\right\}$ is coprime for each $i \in[1 \ldots n]$.

Thm. 3 proves that Algorithm 4 is correct.

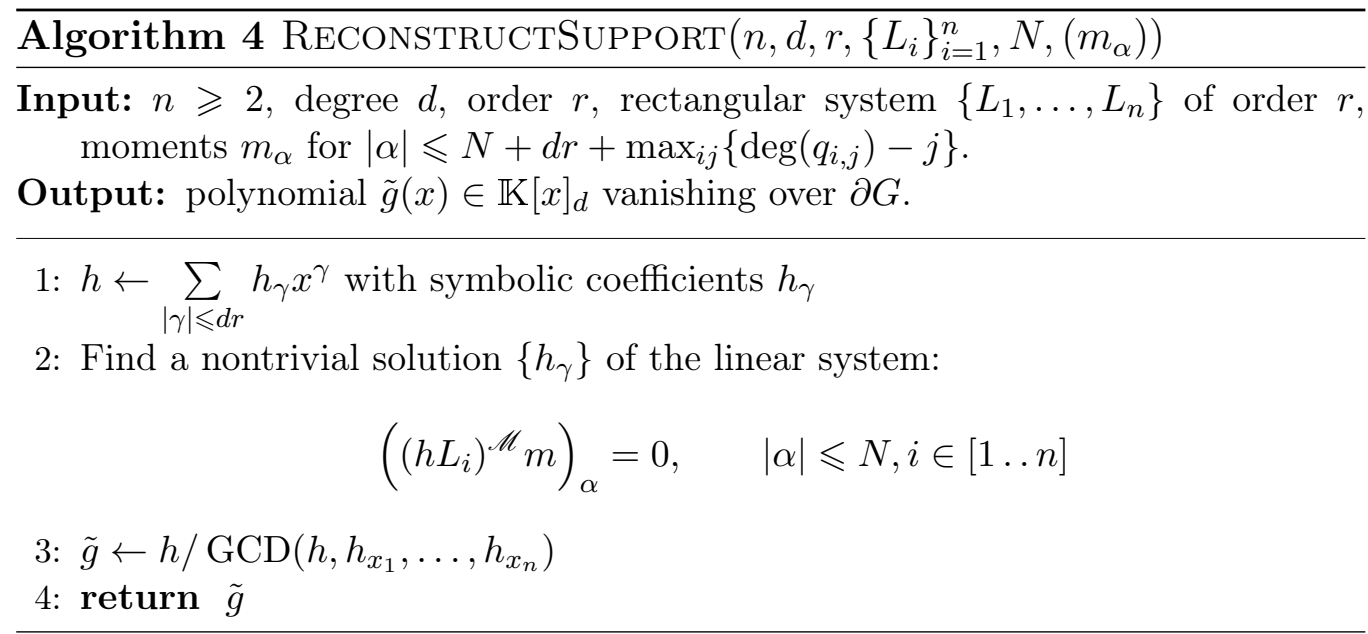

Theorem 3. Let analytic $f$ annihilated by the order $r$ rectangular system $\left\{L_{1}, \ldots, L_{n}\right\}$, and $G$ as in Assumption 1 with $g \in \mathbb{K}[x]$ of degree d. Assume also Assumption 2. Then, for $N \geqslant \hat{N}:=(2 r-1) d+(d-1) b+s$, with $b=r$ $\bmod 2$ and $s=\max \left\{q_{i, r}\right\}, \operatorname{RECONSTRUCTSUpport}\left(n, d, r,\left\{L_{i}\right\}, N,\left(m_{\alpha}\right)\right)$ returns $\tilde{g}=\lambda g$ with $\lambda \in \mathbb{K}^{*}$.

In particular, this proves that when the density is known, the support can be reconstructed using moments up to degree $(3 r-1) d+(d-1) b+s+$ $\max _{i j}\left\{\operatorname{deg}\left(q_{i, j}\right)-j\right\}$.

\footnotetext{
${ }^{1}$ Indeed, if $L_{i}$ has order $r_{i}<r$, then it is replaced by $\partial_{x_{i}}^{r-r_{i}} L_{i}$, which has order $r$ and the same leading polynomial coefficient.
} 
Proof. First $h=g^{r}$ satisfies the linear system line 2 since $g^{r} L_{i} \in \mathfrak{A} \mathfrak{n} \mathfrak{n}\left(f \mathbb{1}_{G}\right)$ by Prop. 4. Let $h$ be any nontrivial solution, then $\left\langle h L_{i}\left(f \mathbb{1}_{G}\right), \varphi\right\rangle=0$ for all $i \in[1 \ldots n]$ and $\varphi \in \mathbb{K}[x]_{N}$. Using Prop. 3 combined with $L_{i} f=0$, we get

$$
\int_{\partial G} \mathcal{L}_{L_{i}}(f, h \varphi) \cdot \boldsymbol{n} \mathrm{d} S=0, \quad i \in[1 \ldots n], \varphi \in \mathbb{K}[x]_{N} .
$$

Since $L_{i}$ involves derivatives only in $x_{i}$, we have $\mathcal{L}_{L_{i}}(f, h \varphi)=\mathcal{L}_{L_{i}, i}(f, h \varphi) \boldsymbol{e}_{\boldsymbol{i}}$, with the Lagrange bilinear concomitant [8]:

$$
\begin{aligned}
\mathcal{L}_{L_{i}, i}(f, & h \varphi)=f\left[q_{i, 1} h \varphi-\partial_{x_{i}}\left(q_{i, 2} h \varphi\right)+\cdots+(-1)^{r-1} \partial_{x_{i}}^{r-1}\left(q_{i, r} h \varphi\right)\right] \\
& +\partial_{x_{i}}(f)\left[q_{i, 2} h \varphi-\partial_{x_{i}}\left(q_{i, 3} h \varphi\right)+\cdots+(-1)^{r-2} \partial_{x_{i}}^{r-2}\left(q_{i, r} h \varphi\right)\right] \\
& +\ldots \\
& +\partial_{x_{i}}^{r-1}(f) q_{i, r} h \varphi .
\end{aligned}
$$

We prove $h=\lambda g^{r}$ for some $\lambda \in \mathbb{K}^{*}$ by induction for $k$ from 0 to $r$, showing $h=g^{k} h_{k}$ with $h_{k} \in \mathbb{R}[x]_{(r-k) d}$. Of course this is true for $k=0$ with $h_{0}=h$. Now suppose that $h=g^{k} h_{k}$ for some $k<r$. Then let

$$
\varphi=q_{i, r} h_{k} g^{r-1-k} g_{x_{i}}^{b} \in \mathbb{K}[x]_{(2 r-2 k-1) d+(d-1) b+s} \subseteq \mathbb{K}[x]_{N},
$$

Since $h \varphi$ is a multiple of $g^{r-1}$, all the terms in 20 are multiples of $g$ (hence they vanish over $\partial G$ ), except for the derivative of order $r-1$, which we can write as

$$
\partial_{x_{i}}^{r-1}\left(q_{i, r} h \varphi\right)=(r-1) ! g_{x_{i}}^{r-1+b} q_{i, r}^{2} h_{k}^{2}+\ell(x) g(x), \quad \ell(x) \in \mathbb{K}[x] .
$$

Therefore, integrating $\mathcal{L}_{L_{i}, i}(f, h \varphi) \boldsymbol{e}_{\boldsymbol{i}} \cdot \boldsymbol{n} \mathrm{d} S$ over $\partial G$ gives

$$
\int_{\partial G}\left(g_{x_{i}^{2}}^{\frac{r+b}{2}} q_{i, r} h_{k}\right)^{2} \frac{f}{\|\nabla g\|} \mathrm{d} S=0,
$$

implying that the squared polynomial in the integrand vanishes over $\partial G$, hence is a multiple of $g$. But $g$ and $q_{i, r}$ are coprime by Assumption 2, so that $g$ divides $h_{k} g_{x_{i}}$, for all $i \in[1 \ldots n]$. Finally, since $\left\{g, g_{x_{1}}, \ldots, g_{x_{n}}\right\}$ is a coprime family, $g$ divides $h_{k}$, giving $h_{k}=g h_{k+1}$.

Now that $h=\lambda g^{r}, \operatorname{GCD}\left(h, h_{x_{1}}, \ldots, h_{x_{n}}\right)=g^{r-1}$ (again since $\left\{g, g_{x_{1}}, \ldots, g_{x_{n}}\right\}$ is coprime), so $\tilde{g}=\lambda g$.

\section{Examples and Conclusion}

We now exemplify our methods in the two dimensional case, with respect to Lebesgue and restricted Gaussian measures 2 . The implementation uses

\footnotetext{
${ }^{2}$ The corresponding code will be available at http://homepages.laas.fr/fbrehard/ HolonomicMomentProblem
} 
OreAlgebra and OreGroebnerBasis routines from the HolonomicFunctions library [11]. The exactly computed moments $m_{i j}$ (obtained from the recurrences given by Algorithm 1 together with closed-form initial conditions, when possible) are truncated to $\tilde{m}_{i j}$, s.t. $\left\lfloor-\log _{10} \frac{m_{i, j}-\tilde{m}_{i j}}{m_{i j}}\right\rfloor=\varepsilon$ i.e., $\varepsilon$ represents the number of correct digits of $\tilde{m}_{i j}$.

In a second time, Algorithm 2 solves the inverse problem given the approximate $\tilde{m}_{i j}$. For numerically solving the resulting overdetermined systems of linear equations, we employ a Least Mean Squares method of Mathematica.

Example 3 (Algebraic Support, Lebesgue measure). Consider the moments $m_{i j}=\int_{G} x^{i} y^{j} d x d y$, with respect to the Lebesgue measure, with $G$ depicted with the checkered pattern in Figure 1.

(i) Direct problem: Given $g=\left(x^{2}+y^{2}-1\right)\left(x^{2}+y^{2}-9\right)\left(x^{2}+(y-\right.$ $\left.2)^{2}-1\right)\left((x-2)^{2}+y^{2}-1\right)$, which vanishes on $\partial G$, and $\mathfrak{A} \mathfrak{n} \mathfrak{n}\{1\}=\left\{\partial_{x}, \partial_{y}\right\}$, Algorithm 1 returns a Gröbner basis with 9 generators and with 36 monomials under the staircase: $\left\{S_{i}^{k} S_{j}^{l}, k, l \in \mathbb{N}, k+l \leqslant 7\right\}$.

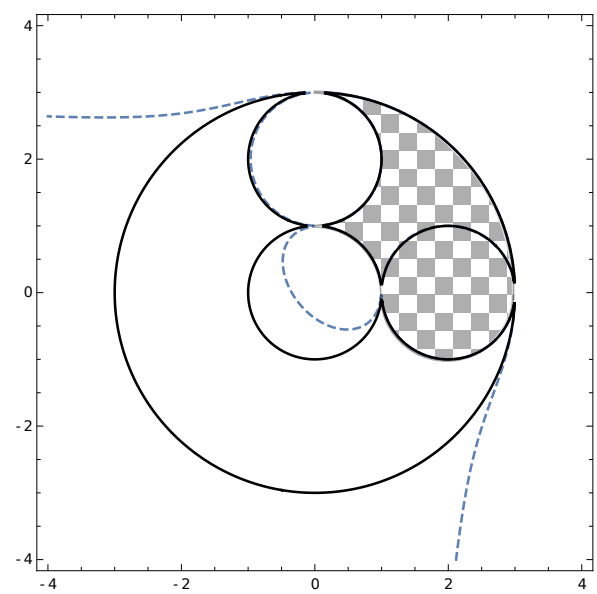

(a)

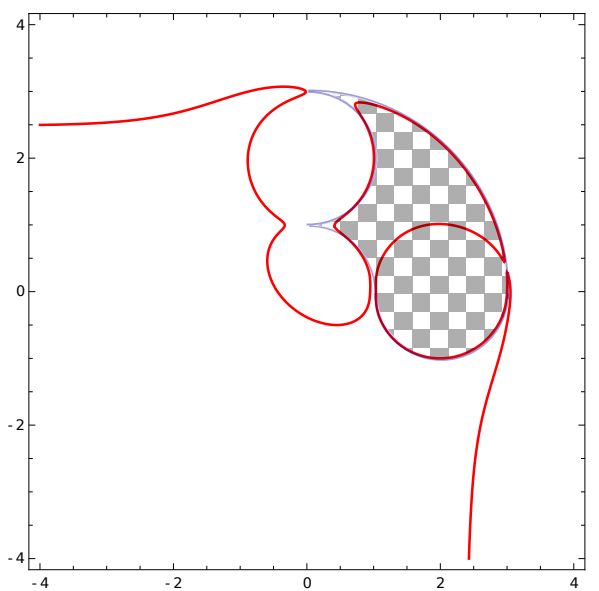

(b)

Figure 1: (a) $G$ in checkered pattern, together with $\partial G$ in black. For $\varepsilon>4$ : reconstructed and original boundary cannot be distinguished at this scale; in dashedblue, $\varepsilon=4$, while in red (b) $\varepsilon=2$.

(ii) Inverse problem: Suppose now given a finite number of numerically computed moments $\tilde{m}_{i j}$ of the Lebesgue measure with unknown support $G$. The goal is to reconstruct $g=\sum_{i+j \leqslant 8} g_{i j} x^{i} y^{j}$ which vanishes on $\partial G$. The results of Algorithm 2 called with parameters $\left(2,8,0,22,\left(\tilde{m}_{i j}\right)_{|i+j| \leqslant 29}\right)$ are depicted in Figure 1: the reconstructed boundary cannot be distinguished from the exact 
at the drawing scale, when the moments $\tilde{m}_{i j}$ are given with more than 4 correct digits. When $2 \leqslant \varepsilon \leqslant 4$, the actual geometric boundary of $G$, can still be very well reconstructed, although the algebraic boundary is degraded.

Example 4 (Algebraic Support, Gaussian measure). Consider the moments $m_{i j}=\int_{G} x^{i} y^{j} \exp (p(x, y)) d x d y$. In Figure 2(a), $G$ is checkered and the level curves of $\exp (p(x, y))$ are in dashed.

(i) Direct problem: Given $g=\left(x^{2}-9 / 10\right)^{2}+\left(x^{2}-11 / 10\right)^{2}-1$, which vanishes on $\partial G$, and $f=\exp \left(-x^{2}+x y-y^{2} / 2\right)$, with $\mathfrak{A} \mathfrak{n} \mathfrak{n}\{f\}=\left\{\partial_{x}+\right.$ $\left.2 x-1, \partial_{y}+y-1\right\}$, apply Algorithm 1 to compute a Gröbner basis for the sequence of moments $m_{i j}$. In the same setting as above, a Gröbner basis with 5 generators and with 28 monomials under the staircase is obtained.

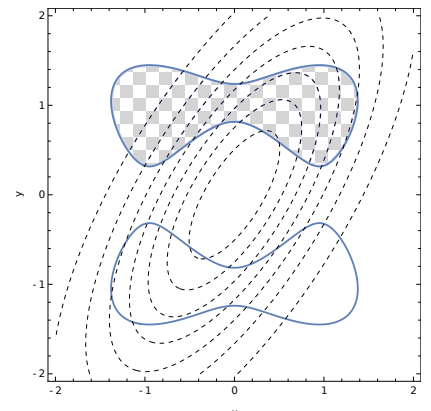

(a)

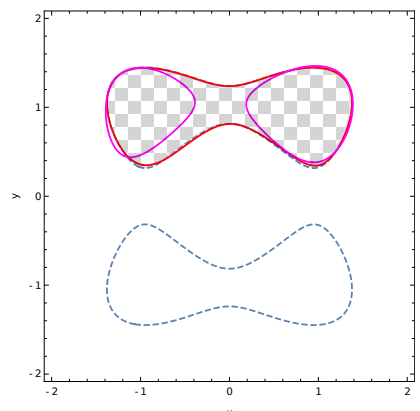

(b)

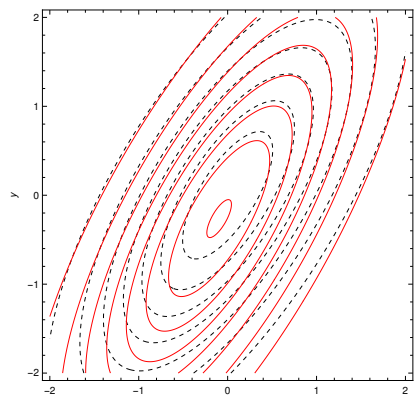

(c)

Figure 2: (a) $G$ in checkered pattern, exact Gaussian level curves in dashed-black, $\partial G$ in blue; (b) When $\varepsilon>8$ : reconstructed and original boundary (in dashed blue) cannot be distinguished at this scale; in red, $\varepsilon=6$, and in magenta for $\varepsilon=4$ digits. (c) Reconstructed Gaussian level curves in red when $\varepsilon=8$; when $\varepsilon>8$, the level curves of exact and reconstructed coincide at this scale.

(ii) Inverse problem: Suppose now given a finite number of numerically computed moments $\tilde{m}_{i j}$, with unknown support $G$ and unknown Gaussian weight. The goal is to reconstruct $g=\sum_{i+j \leqslant 4} g_{i j} x^{i} y^{j}$ which vanishes on $\partial G$, as well as $p=\sum_{i+j \leqslant 2} p_{i j} x^{i} y^{j}$. Algorithm 2 called with parameters $\left(2,4,2,14,\left(\tilde{m}_{i j}\right)_{|i+j| \leqslant 18}\right)$ provides the reconstructed $g$, as depicted in Figure 2(b): the reconstructed boundary cannot be distinguished from the exact at the drawing scale, when $\varepsilon>8$. When $4 \leqslant \varepsilon \leqslant 8$, the actual geometric boundary of $G$, can still be very well reconstructed. Concerning the Gaussian weight, the situation is similar, cf. Figure 2(c).

The examples above are purely academic and even if the proposed method is very robust in these cases, further investigation is needed for the efficient 
numerical implementation of the provided algorithms, in practical higherdimensional applications.

On the theoretical side, this article provides further insight on the question raised in [14] regarding the finite determinateness of a measure. To sum up, provided Assumption 1 and 2 hold, for a measure with compact algebraic support $G$, with $g \in \mathbb{R}[x]_{d}$ vanishing on $\partial G$ and known holonomic density $f$, the moments up to degree $N$ (which only depends on $d$ and the order of a rectangular differential system which annihilates $f$ ) determine in a constructive and robust manner the coefficients of $g$. Thus, this determines in turn all the other moments. When both the density and the support are unknown, a uniform bound $N$ does not exist in general. We provided in this article the solution for the special case of unknown exponential-polynomial density.

Acknowledgement. The research of the third author was funded by the European Research Council (ERC) under the European's Union Horizon 2020 research and innovation program (grant agreement 666981 TAMING).

\section{References}

[1] D. Batenkov. Moment inversion problem for piecewise $D$-finite functions. Inverse Problems, 25(10):105001, 24, 2009.

[2] A. Bostan, F. Chyzak, P. Lairez, and B. Salvy. Generalized hermite reduction, creative telescoping and definite integration of d-finite functions. In Proceedings of International Symposium on Symbolic and Algebraic Computation, New York, USA,2018, pages 95-102, 2018.

[3] F. Chyzak. The ABC of Creative Telescoping: Algorithms, Bounds, Complexity. Memoir of accreditation to supervise research (HDR), Université d'Orsay, Apr. 2014.

[4] P. Ebenfelt, B. Gustafsson, D. Khavinson, and M. Putinar, editors. Quadrature domains and their applications, volume 156 of Operator Theory: Advances and Applications. Birkhäuser Verlag, Basel, 2005.

[5] A. Galligo. Some algorithmic questions on ideals of differential operators. In European Conference on Computer Algebra, pages 413-421. Springer, 1985.

[6] G. H. Golub, P. Milanfar, and J. Varah. A stable numerical method for inverting shape from moments. SIAM Journal on Scientific Computing, 21(4):12221243, 1999.

[7] N. Gravin, J. Lasserre, D. V. Pasechnik, and S. Robins. The inverse moment problem for convex polytopes. Discrete \& Comp. Geometry, 48(3):596-621, 2012. 
[8] E. L. Ince. Ordinary Differential Equations. Dover Publications, New York, 1956.

[9] S. Karlin and W. J. Studden. Tchebycheff systems: With applications in analysis and statistics. Pure and Applied Mathematics, Vol. XV. Interscience Publishers John Wiley \& Sons, 1966.

[10] C. Koutschan. Advanced applications of the holonomic systems approach. $\mathrm{PhD}$ thesis, Research Institute for Symbolic Computation (RISC), Johannes Kepler University, Linz, Austria, 2009.

[11] C. Koutschan. Advanced applications of the holonomic systems approach. ACM Comm. Computer Algebra, 43(3/4):119, 2009.

[12] J. B. Lasserre. Moments, positive polynomials and their applications, volume 1 of Imperial College Press Optimization Series. Imperial College Press, London, 2010.

[13] J. B. Lasserre. Recovering an homogeneous polynomial from moments of its level set. Discrete Comput. Geom., 50(3):673-678, 2013.

[14] J. B. Lasserre and M. Putinar. Algebraic-exponential data recovery from moments. Discrete Comput. Geom., 54(4):993-1012, 2015.

[15] B. Mourrain. Polynomial-exponential decomposition from moments. Found. Comput. Math., 18(6):1435-1492, 2018.

[16] F. Natterer. The mathematics of computerized tomography, volume 32 of Classics in Applied Mathematics. (SIAM), Philadelphia, PA, 2001.

[17] T. Oaku. Algorithms for integrals of holonomic functions over domains defined by polynomial inequalities. J. Symbolic Comput., 50:1-27, 2013.

[18] L. Schwartz. Théorie des distributions. Publications de l'Institut de Mathématique de l'Université de Strasbourg, No. IX-X. Nouvelle édition, entiérement corrigée, refondue et augmentée. Hermann, Paris, 1966.

[19] N. Takayama. An algorithm of constructing the integral of a module - an infinite dimensional analog of gröbner basis. In Proceedings of the International Symposium on Symbolic and Algebraic Computation, Tokyo, Japan, 1990, pages 206-211, 1990.

[20] H. Whitney. Geometric integration theory. Princeton University Press, 1957. 


\section{Appendix}

\section{A.1 Complementary proofs}

Proof of Proposition 11. To prove $(i)$, we use $\partial_{x_{i}} e^{-\mathbf{i} x \cdot z}=-\mathbf{i} z_{i} e^{-\mathbf{i} x \cdot z}$ and $\partial_{z_{i}} e^{-\mathbf{i} x \cdot z}=-\mathbf{i} x_{i} e^{-\mathbf{i} x \cdot z}$ :

$$
\begin{aligned}
\mathscr{F}\{L T\} & =\left\langle T, L^{*}\left(e^{-\mathbf{i} x \cdot z}\right)\right\rangle \\
& =\left\langle T, \sum_{\beta}(-1)^{|\beta|} \partial_{x}^{\beta}\left(q_{\beta}(x) e^{-\mathbf{i} x \cdot z}\right)\right\rangle \quad \text { by definition, } \\
& =\left\langle T, \sum_{\beta}(-1)^{|\beta|} q_{\beta}\left(\mathbf{i} \partial_{z}\right) \partial_{x}^{\beta}\left(e^{-\mathbf{i} x \cdot z}\right)\right\rangle \\
& =\left\langle T, \sum_{\beta}(-1)^{|\beta|} q_{\beta}\left(\mathbf{i} \partial_{z}\right)(-\mathbf{i} z)^{\beta} e^{-\mathbf{i} x \cdot z}\right\rangle \\
& =\left\langle T, L^{\mathscr{F}} e^{-\mathbf{i} x \cdot z}\right\rangle=L^{\mathscr{F} \mathscr{F}\{T\} .}
\end{aligned}
$$

The last equality holds since for any $\mathcal{C}^{\infty}$ function $f(x, z)$,

$$
\begin{aligned}
\left\langle T, z_{i} f(x, z)\right\rangle & =z_{i}\langle T, f(x, z)\rangle, \quad \text { and } \\
\left\langle T, \partial_{z_{i}} f(x, z)\right\rangle & =\left\langle T, \lim _{h \rightarrow 0} \frac{f\left(x, z+h \boldsymbol{e}_{\boldsymbol{i}}\right)-f(x, z)}{h}\right\rangle \\
& =\lim _{h \rightarrow 0} \frac{1}{h}\left(\left\langle T, f\left(x, z+h \boldsymbol{e}_{\boldsymbol{i}}\right)\right\rangle-\langle T, f(x, z)\rangle\right) \\
& =\partial_{z_{i}}\langle T, f(x, z)\rangle,
\end{aligned}
$$

where the commutation of the limit symbol comes from the fact that $f_{z, h}: x \mapsto$ $\frac{f\left(x, z+h \boldsymbol{e}_{\boldsymbol{i}}\right)-f(x, z)}{h}$ converges to $f_{z}: x \mapsto f(x, z)$ for the compact-open topology of $\mathcal{E}$. To prove $(i i)$, one just need to notice that $L^{\mathscr{M}}$ is obtained from $L^{\mathscr{F}}$ using

$$
z_{i} \mapsto \mathbf{i} \alpha_{i} S_{\alpha_{i}}^{-1}, \quad \text { and } \quad \partial_{z_{i}} \mapsto-\mathbf{i} S_{\alpha_{i}}
$$

